PROGRESS REPORT: 1 SEPTEMBER 1990 - 1 MARCH 1992

DOE Document Number DOE/ER/13958-28

\title{
SEMIEMPIRICAL STUDIES OF ATOMIC STRUCTURE
}

U.S. Department of Energy, Fundamental Interactions Branch Division of Chemical Sciences, Office of Basic Energy Sciences

Grant Number DE-FG05-88ER13958

L. J. Curtis

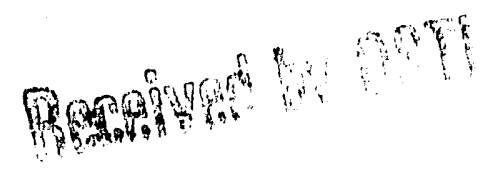

FEB $2: 1992$

Department of Physics and Astronomy

University of Toledo

2801 W. Bancroft Street

Toledo, OH 43606

tel. (419) $53 \%-2341$

fax (419) 537-2723

bitnet fac0283 @ uoft01

email: 1jc @ utphya.phya.utoledo.edu

\section{DISCL “ IMER}

This report was prepared as an account of work sponsored by an agency of the United States This repon was prepared as an acoutes Government nor any agency thereof, nor any of their Government. Neither the United States Government nor any agency lhe liability or responsiemployees, makes any warranty, express or implied, or assumes any legal liabilitus, product, or bility for the accuracy, completeness, or usefulness of any information, apparatus, product, or process disclosed, or represents that its use would not infringe privately owned rights. Reference herein to any specific commercial product, process, or service by trade name, trademark, manufacturer, or otherwise does not necessarily constitute or imply ils endorement, The views mendation, or favoring by the United States Government or any agency thect those of the and opinions of authors expressed 1 rein do not necessarily state or reflect those of the United States Government or any agency thereof. 


\section{SCOPE OF INVESTIGATIONS}

The energy level structure transition probabilities, and general spectroscopic properties of high ly-ionized many-electron systems are studied through the combined use of sensitive semiempirical data systematizations, selected precision experimental measurements, and specialized theoretical computations. Measurements are made primarily through the use of fast ion beam excitation methods, which are combined with available data from laser- and tokamakproduced plasmas, astrophysical sources, and conventional light sources. The experimental studies are strengthened through large scale ab initio calculations. Large blocks of data are predictively systematized and parametrized along isoelectronic, homologous, isoionic, Rydberg, and yrast series, to provide a comprehensive and reliable data base.

\section{SIGNIFICANT RESULTS}

During the period from 1 September 1990 to 1 March 1992, work done as part of this project has resulted in twelve published papers [1-12]. Some of the projects carried out during the past year include: isoelectronic systematizations of two and three electron systems which combine new and existi:; lifetime measurements with intermediate ce upling information deduced from observed energy level data; accurate semiempirical extrapolations and interpolation of energy levels, ionization potentials, and core polarizabilities for all ions in the $\mathrm{Li}$ sequence, and the use of these data to deduce transition probabilities by the semiempirical CAHS method; high precision relative lifetime measurerents in the $\mathrm{Li}$ sequence by a new technique utilizing a position sensitive det:ctor; and studies of cancellation effects in transition probabilities which explain recently measured anomalies in the Au isoelectronic sequence. An extensive review of important aspects of this work is given in Ref[1], and brief descriptions of the selected the results reported in Refs[1-12] are given below.

\section{Mixing Angle Formulations which combine Spectroscopic and Lifetime Data}

In studies of $n s^{2}-n s n^{p} \mathrm{p}$ and $n p^{6}-n p^{5} n$ 's transitions in alkaline-earthlike and inert-gaslike isoelectronic sequences we have developed an empirical data reduction that treats measured transition probabilities for the resonance and intercombination lines as conjugate quantities. The reduction is formulated through a singlet-triplet mixing angle $\vartheta$ deduced from measured spectroscopic energy level data, and yields an effective generalized line strength that has a regular and slowly varying isoelectronic behavior. This regularity has been observed to persist even in the presence of strong perturbations, and it can be linearized through simple screening parametrizations that permit quantitative interpolation, extrapolation and smoothing. The total effective line strength is defined as the sum of the line strengths of the resonance and intercombination transitions, which can be obtained from either individual line strength by dividing by the appropriate empirical quantity $\sin ^{2} \theta$ or $\cos ^{2} \vartheta$. The combined use of resonance and intercombination lines to specify a generalized line strength permits data over a wide range of charge states to be merged. Time-of-flight methods are limited to lifetimes that produce a measurable decay length, and of ten the intercombination transitions are unmeasurably long-lived at low $\mathrm{Z}$ while the resonance transitions are unmeasurable short-lived at high $\mathrm{Z}$. Using our own lifetime measurements as well as those of other groups, predictive isoelectronic studies have been completed for a variety of systems. These include the strong low lying intrashell resonance and intercombination lines for the $2 s^{2}-2 s 2 p, 3 s^{2}-3 s 3 p$, and $4 s^{2}-4 s 4 p$ transitions in the $\mathrm{Be}[3], \mathrm{Mg}[3]$, and $\mathrm{Zn} \mathrm{[12]} \mathrm{isoelectronic} \mathrm{sequences.} \mathrm{In}$ 
all three cases the reduced line strength is found to be a nearly linear function of the reciprocal screened charge, and the slopes and intercepts exhibit a homologous regularity.

The singlet-triplet mixing angles described above pertain to systems in which only two levels are mixed by intermediate coupling. The method can be extended to systems of arbitrary complexity, although this requires the diagonalization of a matrix larger than $2 \times 2$. For the $3 \times 3$ matrix which occurs for an $n \mathrm{p}^{3}$ configuration, specification of the doublet-quartet mixing coefficients requires the solution of a cubic secular equation

$$
\lambda^{3}+a_{2} \lambda^{2}+a_{1} \lambda+a_{0}=0 \text {. }
$$

Although the explicit solutions for the energy eigenvalues $E_{1}$ as a function of the coefficients al (which involve Slater and spin-orbit integrals) are complicated and cumbersome, the inverse solution is quite simple, given by

$$
a_{2}=-E_{1}-E_{2}-E_{3} ; \quad a_{1}=E_{1} E_{2}+E_{2} E_{3}+E_{3} E_{1} ; \quad a_{0}=-E_{1} E_{2} E_{3}
$$

where the energy eigenvalues are spectroscopically measured quantities. The empirical eigenvectors generated in this way can be used to reduce measured transition probabilities to effective transition integrals or vice versa.

This method has been applied [7] to make specific calculations for the $N$ and $P$ sequences.

\section{Cancellation Effects in Isoelectronic Sequences}

Atomic transition moments sometimes exhibit cancellation effects that can cause a normally strong transition to be weak or totally absent in certain observed spectra. One class of cancellation effects can occur within a single-electron radial integral as a result of differential phase shifts in the wave functions due to core polarization and penetration. Cancellation effects in a specific transition probability do not usually lead to a large anomaly in the lifetime of the upper level, since these cancellations occur only for $\Delta n \neq 0$ transitions (which possess multiple decay branches), and $f$ sum rules tend to redistribute the missing oscillator strength among other exit channels. However, a recent lifetime measurement by Pinnington and coworkers in the $\mathrm{Au}$ isoelectronic sequence exhibited an anomaly which we have explained in terms of such a cancellation. They observed that for the $7 \mathrm{p}$ term in $\mathrm{Pb} I V$ the $J=1 / 2$ level has a lifetime six times longer than that of the $J=3 / 2$. We have developed methods to systematically study these radial integral cancellations and to predict their occurrence by simple graphical methods, and have applied this approach [6] to demonstrate that this result is a consequence of a nearly complete cancellation in the $6 s-7 p$ radial transition moment for the $J=1 / 2$ level, coupled with fortuitous $\lambda^{3}$ factors which suppress the decay to $7 \mathrm{~s}, 6 \mathrm{~d}$, and $6 \mathrm{~s}^{2}$.

\section{Experimental and Computational Studies of Lifetimes in the Li Sequence}

We have reported [8] lifetime predictions for the $2 \mathrm{p}$ levels in the $\mathrm{Li}$ iscelectronic sequence for $Z=3-92$ using the semiempirical Coulomb approximation with a Hartree-Slater model core (CAHS). This extends our earlier studies of the $\mathrm{Na}$ and $\mathrm{Cu}$ sequences, and the results agree well with critically selected experimental lifetime measurements. These calculations require as inputs accurate values for the experimental excitation energies, the ionization potentials, and the core polarizabilities, and we have also reported a comprehensive and reliable data base for the se quantities using a combination of semiempirical parametrizations of spectroscopic measurements and ab initio calculations. 
We have also complemented these computational studies with measurements [9] of lifetimes in Li-like silicon atoms using multiplexed detection of a fast ion beam source. This permitted simultaneous measurements of the two fine structure components of the resonance transition, which yielded a precise determina:ion of the lifetine ratio. This provides a sensitive test of deviations from the $\lambda^{3}$ nonrelativistic scaling. These measurements were also accompanied by new relativistic many-body calculations of the lifetimes of the $2 p$ fine structure states in Si XII.

\section{BIBLIOGRAPHY OF PUBLICATIONS PRODUCED ON THIS PROJECT 1 September 1990 - 1 March 1992}

1. L.J. Curtis and I. Martinson, "Lifetime Measurements in Multiply Ionized Atoms," Comments in Atomic and Molecular Physics 24, 213-33 (1990). [Document DOE/ER/13958-16]

2. L.J. Curtis, "Classical Mmenonic Approach for Obtaining Hydrogenic Expectation Values of $r^{P}$," Phys. Rev. A 43, 568-9 (1991). [Document DOE/ER/13958-17]

3. L.J. Curtis, "Isoelectronic Smoothing of Line Strengths in Intermediate Coupling," Physica Scripta 43, 137-43 (1991). [Document. DOE/ER/13958-18]

4. R.R. Haar, L.J. Curtis, T.J. Kvale, D.J. Beideck, I. Martinson and R. Hellborg, "Lifetimes of Excited Levels in Neutral Carbon," Astronomy and Astrophysics 243, 321-6 (1991). [Document DOE/ER/13958-19]

5. R. Hellborg, I. Martinson, S.T. Maniak, R.E. Irving, R.R. Haar, L.J. Curtis and D.J. Beideck, "Variations of Carbon Foil Lifetimes under Bombardment by $\mathrm{N}^{+}, \mathrm{Ne}^{+}, \mathrm{Ar}^{+}$and $\mathrm{Zn}^{+}$Ions," Physica Scripta 43, 262-5 (1991). [Document DOE/ER/13958-20]

6. L.J. Curtis, "Cancellation Effects in Alkalilike Doublets," Can. J. Phys. 69, 668-70 (1991). [Document DOE/ER/13958-21]

7. L.J. Curtis, Z.B. Rudzikas, and D.G. Ellis, "Empirical Determination of Intermediate Coupling Amplitudes and Transition Rates from Spectroscopic Data," Phys. Rev. A 44, 776-9 (1991). [Document DOE/ER/13958-22]

8. C.E. Theodosiou, L.J. Curtis, and M. El-Mekki, "Calculations of $2 p$ Lifetimes in the Li Sequence," Phys. Rev. A 44, 7144-57 (1991). [Document DOE/ER/13958-23]

9. A.E. Livingston, F.G. Serpa, A.S. Zacarias, L.J. Curtis, H.G. Berry and S.A.Blundell, "Lifetime Measurements in Highly-Ionized Silicon, Phys. Rev. A 44, 7820-2 (1991). [Document DOE/ER/13958-24]

10. R.W. Dunford, C.J. Liu, J. Last, N. Berrah-Mansour, R. Vondrasek, D.A. Church, and L.J. Curtis, "Direct Observation of Hyperfine Quenching of the 2 Po Level in Heliumlike Nickel," Phys. Rev. A. 44, 764-7 (1991). [Document DOE/ER/13958-25]

11. R.W. Dunford, H.G. Berry, D.A. Church, T.P. Dineen, M. Hass, C.J. Liu, N. Berrah-Mansour, R.C. Pardo, M.L.A. Raphaelian, L. Young, B.J. Zabransky, and L.J. Curtis, "Precision Spectroscopic Measurements in Few-Electron Ions," Z. Physik D 21, S13-16 (1991). [Document DOE/ER/13958-26]

12. L.J. Curtis, "Empirical Lifetimes for $4 s 4 \mathrm{p}{ }^{1,3} \mathrm{P}$ Levels in the $\mathrm{Zn}$ Isoelectronic Sequence," J. Opt. Soc. Am. B 9, 5-9 (1991).

[Document DOE/ER/13958-27] 

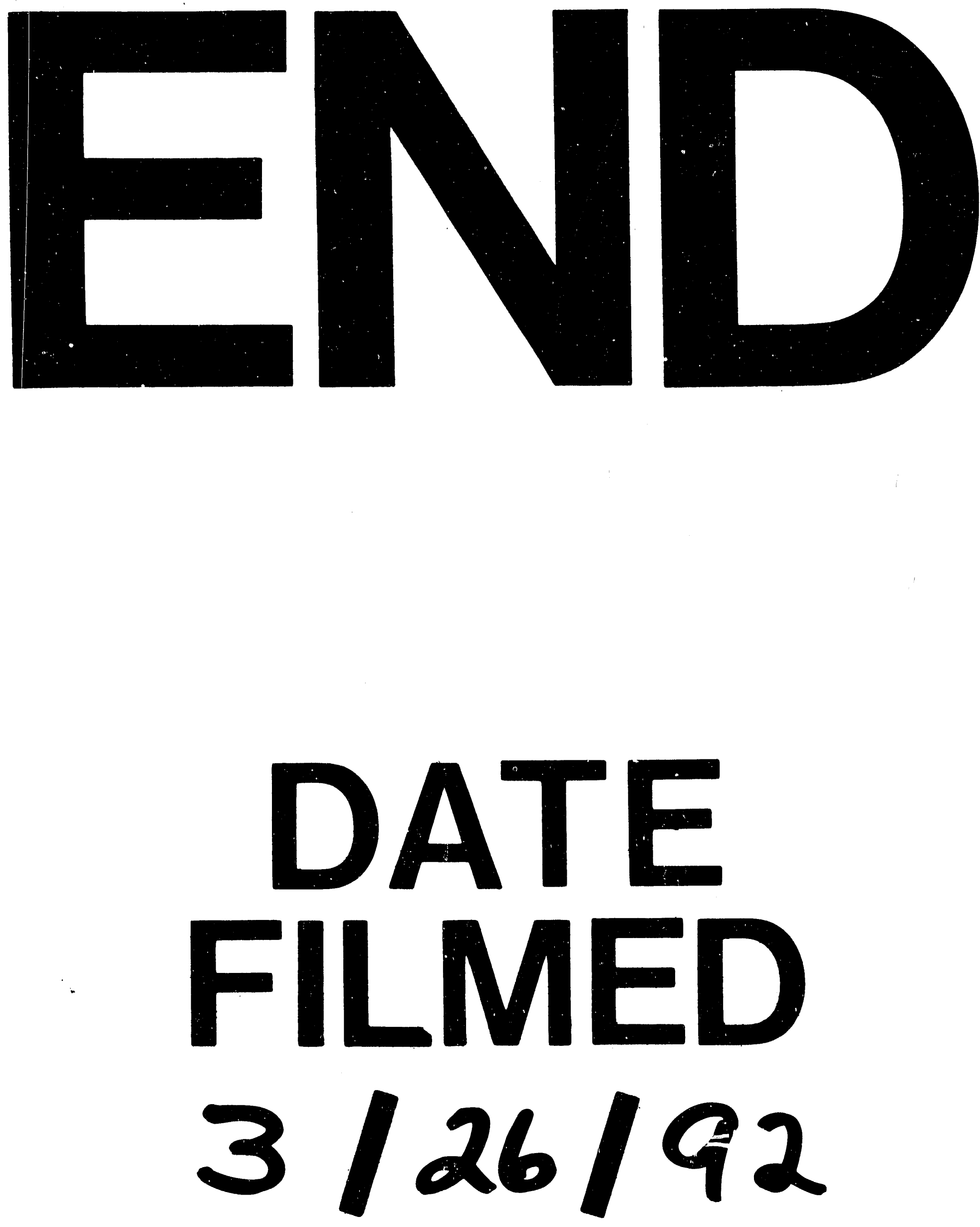
